\title{
Generalization of the Riemann method for the trunk gas pipelines considering the quadratic law of resistance
}

\author{
Ismatulla Khujayev ${ }^{1}$, Sarvarbek Akhmadjonov ${ }^{2 *}$, and Alisher Ismailov ${ }^{3}$ \\ ${ }^{1}$ Institute of Mechanics and Seismic Resistance M.T.Urazbaev Academy of Sciences of the Republic \\ of Uzbekistan, Tashkent, Uzbekistan \\ ${ }^{2}$ Andijan Machine-building Institute, Andijan, Uzbekistan \\ ${ }^{3}$ Tashkent Institute of Textile and Light Industry, Tashkent, Uzbekistan
}

\begin{abstract}
A mathematical model of the problem of pulse propagation in a semi-infinite gas pipeline was developed by expressing the pressure drop by the quadratic law of resistance and the local component of the gas inertia force by the law of conservation of momentum, using the law of conservation of mass in a one-dimensional statement. The model repeats the Riemann problem but takes into account the frictional resistance force. Using an auxiliary function in the form of the natural logarithm of the reduced density, and gauge functions, and certain simplifications, an equation for the reference solution of the problem in terms of gas velocity was derived and solved. For the analytical solution of the problem on gas velocity, the Riemann solution was used, and a refined analytical solution was obtained considering the quadratic law of resistance for the calculation of the perturbed and non-perturbed subdomains.
\end{abstract}

\section{Introduction}

Trunk gas pipelines are the main part of the gas transportation system [1]. The greater share of energy costs in pipeline gas transportation falls on this part of the system. The characteristics of the pipeline network and the set technological tasks are the determining factors for the operating mode of other equipment of the system. In this regard, it is important to ensure the ability to calculate the operating mode of the trunk gas pipeline with a sufficient degree of accuracy. At the same time, consideration should be given to all basic factors affecting the operation of the trunk gas pipelines. Despite the seeming simplicity of the gas pipeline design, complex processes of motion, friction, interaction with gravity forces, internal and external heat transfer take place in it. These processes, as a rule, change over time; that is, they are non-stationary processes.

In [2], a method is proposed to determine the boundaries of the preferred application of a stationary model and the model that describes the non-stationary process of the gas pipeline operation. The study in [3] is devoted to the modeling and numerical calculation of

${ }^{*}$ Corresponding author: s.axmadjonov1990@mail.ru 
the network of the gas-distribution pipelines with special emphasis on the elementary sections of the gas pipeline. In [4], the problems of optimal control of the gas flow in the pipeline network are considered. Quasi-one-dimensional equations are constructed from the complete nonlinear isothermal Euler gas equations. The problem of optimal control in the gas pipeline network is formulated, and its time discretization is performed. In [5], a solution to the actual applied problem of gas quality control is proposed for an extended multilayer-insulated underwater high-pressure gas pipeline under non-stationary nonisothermal operation. A mathematical model and an algorithm for calculating the composition, pressure, velocity, and temperature of a non-stationary non-isothermal gas flow under high operating pressures were developed. A computational experiment was conducted using the developed model.

In [6], a mathematical model of the transition process in gas pipelines and networks is proposed. Modeling is based on the transfer function introduction and the finite volume method. Nonlinear transfer functions are constructed for various types of boundary conditions. In [7], the possibilities of applying the results of mathematical modeling of trunk pipelines to the problems of preliminary calculation of technological and design parameters of natural gas transportation sections are considered.

Simple analytical formulas were obtained to determine the mass flow rate, pressure, and supercompressibility coefficient [8]. But the distribution of the mass flow rate of gas between parallel threads with a variable gas supercompressibility coefficient occurs as it was in the case of a constant value of the gas supercompressibility coefficient. The difference lies in the definition of the input and output pressure values, i.e., when solving transcendental equations.

According to the canons of the turbulence theory, large eddies present in a turbulent flow, by a cascade transition, come to such a size of eddies (Kolmogorov scale), which, further decaying, go to thermal energy. In work [9], a check was made of the position often used in pipeline hydraulics. The work of friction forces produced when a real gas moves through a gas pipeline is completely converted into thermal energy. Using the integral definition of the Clausius entropy, it is shown that this thesis is confirmed with an accuracy acceptable for engineering applications as applied to the one-dimensional formulation of the problem of determining the longitudinal temperature field of gas. From the point of view of the reliability of the pipeline network, resonance phenomena are of great importance. In particular, a decrease in the inlet pressure or flow to the blower can lead to a surge phenomenon, which is fraught with danger due to the formation of oscillations of the entire pipeline network. And in [10], the case was considered when the piston's oscillation generated vibration at one end of the pipeline.

The article [11] presents the results of a study of a gas pipeline under conditions of heat transfer with soil. According to a simplified heat transfer model, stable, one-dimensional non-stationary, and two-dimensional non-stationary thermal state of gas during heat exchange between the pipe wall and the ground according to a simplified heat transfer model are studied. The influence of rapid changes in the mass flow rate of gas and temperature at the inlet to the pipeline is considered. The presented case is typical for export gas pipelines containing offshore and in-depth sections along the route. The results are compared with experimental data from an existing export gas pipeline.

Below we consider a problem when, at $x=0$, the boundary condition is set, and at $t=0$, conditions are set in the form of path changes in the gas velocity and its first-time derivative. The considered solution region is the left semi-axis $x(x \geq 0)$. A solution concerning the gas velocity is obtained, which, as $\varepsilon \rightarrow 0$, turns to the well-known Riemann solution. 


\section{Materials and Methods}

Ignoring the convective component of the gas inertia force and the path inclination from the horizon, the quasi-one-dimensional equations of conservation of momentum and mass have the following form $[12,13]$ :

$$
\left\{\begin{array}{l}
-\frac{\partial p}{\partial x}=\rho \frac{\partial u}{\partial t}+\varepsilon \rho u^{2} \\
-\frac{\partial \rho}{\partial t}=\frac{\partial \rho u}{\partial x}
\end{array}\right.
$$

here $x, t$ are the distance and time; $u, p, \rho$ are the average values of velocity, pressure, and density of gas in the section $x$ at the time point $t ; \varepsilon=\lambda / 2 D$ is the parameter of resistance force in the Darcy-Weisbach law; $\lambda, D$ are the friction drag coefficient and pipeline diameter.

In the absence of external disturbances, the boundary value problem can be posed with the following boundary condition

$$
u(0, t)=\mu(t)
$$

with initial conditions

$$
\begin{aligned}
& u(x, 0)=\phi(x), \\
& \frac{\partial u(x, 0)}{\partial t}=\psi(x) .
\end{aligned}
$$

Thus, the problem of the gas-dynamic state of a semi-infinite pipeline $(x \geq 0)$. is solved in this article.

An introduction of the propagation velocity of small disturbances $c=\sqrt{\partial p / \partial \rho}=\sqrt{Z R T}$ in a real gas $(Z, R, T$ are the supercompressibility factor, reduced gas constant, and average gas temperature, respectively) transported through the pipeline and of an auxiliary function

$$
\varphi=\ln \left(\rho / \rho_{*}\right),
$$

where $\rho_{*}$ is the characteristic gas density, and ignoring the term in the second equation, which at low hydrodynamic velocities and small density perturbations is a small value, allows us to write system (1) in the following form:

$$
\frac{\partial u}{\partial t}+c^{2} \frac{\partial \varphi}{\partial x}=-\varepsilon u^{2}, \frac{\partial \varphi}{\partial t}+\frac{\partial u}{\partial x}=0
$$

In numerous literary sources, including textbooks [10], this system is analyzed and solved without the right-hand side in the first equation. 
In some cases, gauge functions are introduced [15].

$$
A=u+c \varphi, \quad B=u-c \varphi .
$$

Then, system (7) takes the form:

$$
\frac{\partial A}{\partial t}+c \frac{\partial A}{\partial x}=-\varepsilon u^{2}, \frac{\partial B}{\partial t}-c \frac{\partial B}{\partial x}=-\varepsilon u^{2} .
$$

From this system of equations, for example, an equation relative to velocity $u(x, t)=A(\eta, t)+B(\xi, t)$, can be derived

$$
\frac{d u}{u^{2}}=-\frac{\varepsilon d x}{c}, \frac{d x}{d t}=c
$$

where $u^{0}=\left.u(\eta, \xi)\right|_{\varepsilon=0}$ is the solution to the homogeneous system (1), i.e., at $\varepsilon=0$.

Hence, the reference solution to the problem is found as

$$
u=\frac{u^{0}}{1+\frac{\varepsilon x}{c} u^{0}}
$$

From this solution of the problem, the general pattern follows in the approximation of a long pipeline: the solution of the problem considering friction is less than the problem's solution without considering friction.

Now we determine the value of $u^{0}$.

Let us turn to the well-known solution of the Cauchy problem from mathematical physics [16]:

$$
u^{0}(x, t)=\frac{\Phi(x+c t)-\Phi(x-c t)}{2}+\frac{1}{2 \pi} \int_{x-c t}^{x+c t} \Psi(\alpha) d \alpha
$$

where $\Phi$ and $\Psi$ are the values of the unknown and its time derivative at $t=0$.

To have zero value of the unknown at $x=0$, it is necessary to assume that

$$
\Phi(x)=\left\{\begin{array}{l}
\phi(x) \text { at } \quad x>0 \\
-\phi(x) \text { at } \quad x<0
\end{array} \text { and } \Psi(x)=\left\{\begin{array}{l}
\psi(x) \text { at } x>0 \\
-\psi(x) \text { at } x<0
\end{array}\right.\right.
$$

In this case $u^{0}(0, t)=0$, and then we can use the condition at $x=0$. In addition, due to the fulfillment of the following conditions

$$
u^{0}(x, 0)=\Phi(x)=\varphi(x)
$$




$$
\frac{\partial u^{0}(x, 0)}{\partial t}=\Psi(x)=\psi(x)
$$

at $x>0$, the solution satisfies two initial conditions.

Then, taking into account the velocity perturbation in section $x=0$, we can write down the solution to the problem as:

$$
u^{0}(x, t)=\left\{\begin{array}{l}
\mu\left(t-\frac{x}{c}\right)+\frac{\phi(x+c t)-\phi(x-c t)}{2}+\frac{1}{2 a} \int_{c t-x}^{x+c t} \psi(\alpha) d \alpha \text { at } x<t c, \\
\frac{\phi(x+c t)-\phi(x-c t)}{2}+\frac{1}{2 a} \int_{x-c t}^{x+c t} \psi(\alpha) d \alpha \text { at } x>t c .
\end{array}\right.
$$

Substitution of $u^{0}$ into equation (9) leads to the result for the gas velocity:

$$
u(x, t)=\left\{\begin{array}{l}
\frac{\mu\left(t-\frac{x}{c}\right)+\frac{1}{2}[\phi(x+c t)-\phi(x-c t)]+\frac{1}{2} \int_{c t-x}^{x+c t} \psi(\alpha) d \alpha}{1+\frac{\varepsilon x}{c}\left\{\mu\left(t-\frac{x}{c}\right)+\frac{1}{2}[\phi(x+c t)-\phi(x-c t)]+\frac{1}{2} \int_{c t-x}^{x+c t} \psi(\alpha) d \alpha\right\}} \\
\frac{\frac{1}{2}[\phi(x+c t)-\phi(x-c t)]+\frac{1}{2} \int_{x-c t}^{x+c t} \psi(\alpha) d \alpha}{1+\frac{\varepsilon x}{c}\left\{\frac{1}{2}[\phi(x+c t)-\phi(x-c t)]+\frac{1}{2} \int_{x-c t}^{x+c t} \psi(\alpha) d \alpha\right\}} \text { at } x>t c .
\end{array} \text { at } x \leq t c,\right.
$$

Thus, an analytical solution to the formulated problem was obtained for the hydrodynamic gas velocity.

This solution generalizes the known solution of the Riemann problem taking into account the quadratic law of resistance in a gas pipeline. Indeed, if we assume that $\varepsilon=0$, then a classical Riemann solution [14] on a unilateral wave is obtained.

To present the complete picture, it is necessary to find a solution to the pressure problem, for which one can turn to the numerical method.

The transition to discrete coordinates is performed so that the pulse jump falls on the calculated node. This choice of discrete coordinate steps makes it possible to determine the value of the pressure jump at the shock front. To confirm this statement, let us turn to the next problem.

We assume that before the onset of disturbances, the gas is at rest, and at $t=0$ in the inlet to the section, the gas velocity instantly increases to $U$ and then, this constant value of velocity in the inlet remains. Accordingly, the initial pressure value and the pressure value in the unperturbed region is $P$. Following this, the initial value of the density and the value of the gas density in the unperturbed region is $\rho_{0}=P / c^{2}$, i.e., $\rho_{0}$ can be taken for the characteristic gas density. The auxiliary function has a value of $\ln \frac{\rho}{\rho_{0}}=\ln \frac{\rho c^{2}}{P}$. In the unperturbed calculation zone, we have $u=0, \varphi=0$.

Let us assume that the pulse wave reaches the node $n$ in time, which corresponds to the coordinate $x=n h$. The gas velocity at this point is 


$$
u_{n}^{n}=U /\left(1+\frac{\varepsilon n h}{c} U\right)
$$

and at the nodes following the disturbance front along $x$, the gas is at rest, in particular: $u_{n+1}^{n}=0$.

Then from the second equation of system (6), it follows that

$$
\frac{\varphi_{n}^{n}-\varphi_{n}^{n-1}}{\tau}=-\frac{u_{n+1}^{n}-u_{n}^{n}}{h}
$$

Here we used the backward pattern in time and the forward pattern along the $\mathrm{x}$ coordinate. Since at $i>n$ the gas is at rest, the equations $u_{n+1}^{n}=0, \varphi_{n}^{n-1}=0$ hold. So, $\varphi_{n}^{n}=\frac{\tau}{h} u_{n}^{n}$ it follows from the last finite difference equation. Considering $\tau=h / c$, we obtain

$$
\ln \frac{\rho_{n}^{n}}{\rho_{0}}=\ln \frac{p_{n}^{n}}{P}=\frac{u_{n}^{n}}{c}
$$

By potentiating these relations, we obtain the density values

$$
\rho_{n}^{n}=\rho_{0} \exp \left(\frac{U}{c+\varepsilon n h U}\right)
$$

and the pressure values

$$
p_{n}^{n}=\operatorname{Pexp}\left(\frac{U}{c+\varepsilon n h U}\right)
$$

on the disturbance front for the $n$-th time step.

As $x^{*}=n h$ is the coordinate of the wave front, the last relations can be written in the following form

$$
u\left(x^{*}\right)=\frac{c U}{c+\varepsilon x^{*} U}, \quad \rho\left(x^{*}\right)=\rho_{0} \exp \left(\frac{U}{c+\varepsilon x^{*} U}\right), p\left(x^{*}\right)=P \exp \left(\frac{U}{c+\varepsilon x^{*} U}\right)
$$

From the last formula, it follows that the highest-pressure value is

$$
p_{0}^{*}=P \exp (U / c)
$$

that is, $\exp (U / c)$-times jump is expected at the beginning of the process, and then, as the disturbance propagates, the pressure jump decreases. 
Taking into account the obtained value of $\varphi_{n}^{n}$ at the disturbance front and the known values of $u_{i}^{n}$ and $u_{i}^{n-1}$, we turn to the first equation of system (6), represented in a discrete form as:

$$
\frac{\varphi_{i+1}^{n}-\varphi_{i}^{n}}{h}=-\frac{1}{c^{2}}\left[\frac{u_{i+1}^{n}-u_{i+1}^{n-1}}{\tau}+\varepsilon\left(u_{i+1}^{n}\right)^{2}\right]
$$

Here it is taken into account that the pressure change occurs against the direction of pulse wave propagation.

The last finite difference equation implies the recurrence formula

$$
\varphi_{i}^{n}=\varphi_{i+1}^{n}+\frac{h}{c^{2}}\left[\frac{u_{i+1}^{n}-u_{i+1}^{n-1}}{\tau}+\varepsilon\left(u_{i+1}^{n}\right)^{2}\right]
$$

which allows us to calculate the values of the auxiliary function at $i=n-1 . .0$.

Based on the value of $u_{n}^{n}$ and dependence

$$
\frac{\partial \varphi}{\partial x}=-\frac{\varepsilon}{c^{2}} u^{2}=U \frac{d}{d y}\left(\frac{1}{y}\right)
$$

where the designation $y=c+\varepsilon x U$ was introduced, the value of the auxiliary function can be analytically determined as

$$
\varphi(x)=\varphi\left(x^{*}\right)+\frac{1}{c+\varepsilon x U}-\frac{1}{c+\varepsilon x^{*} U} .
$$

With $u_{i}^{n}$ and $\varphi_{i}^{n}$ the values of $\rho_{i}^{n}, p_{i}^{n}$ and $M_{i}^{n}=f \rho_{i}^{n} u_{i}^{n}$ are determined (mass flow $f=\pi D^{2} / 4$ is the cross-sectional area of the pipeline).

\section{Results and Discussion}

A computational experiment was conducted for a pipeline of a diameter of $1 \mathrm{~m}$, a resistance coefficient of 0.01 at $\varepsilon=0.005 \mathrm{~m}^{-1}$. At the beginning of the section, the case was considered when the velocity increased from $0 \mathrm{~m} / \mathrm{s}$ to $20 \mathrm{~m} / \mathrm{s}$. The sound propagation velocity was $378.21 \mathrm{~m} / \mathrm{s}$.

The results are presented in the form of contour lines of average velocity and mass flow rate. The isobar field obtained is similar to the one in Figure 1. 


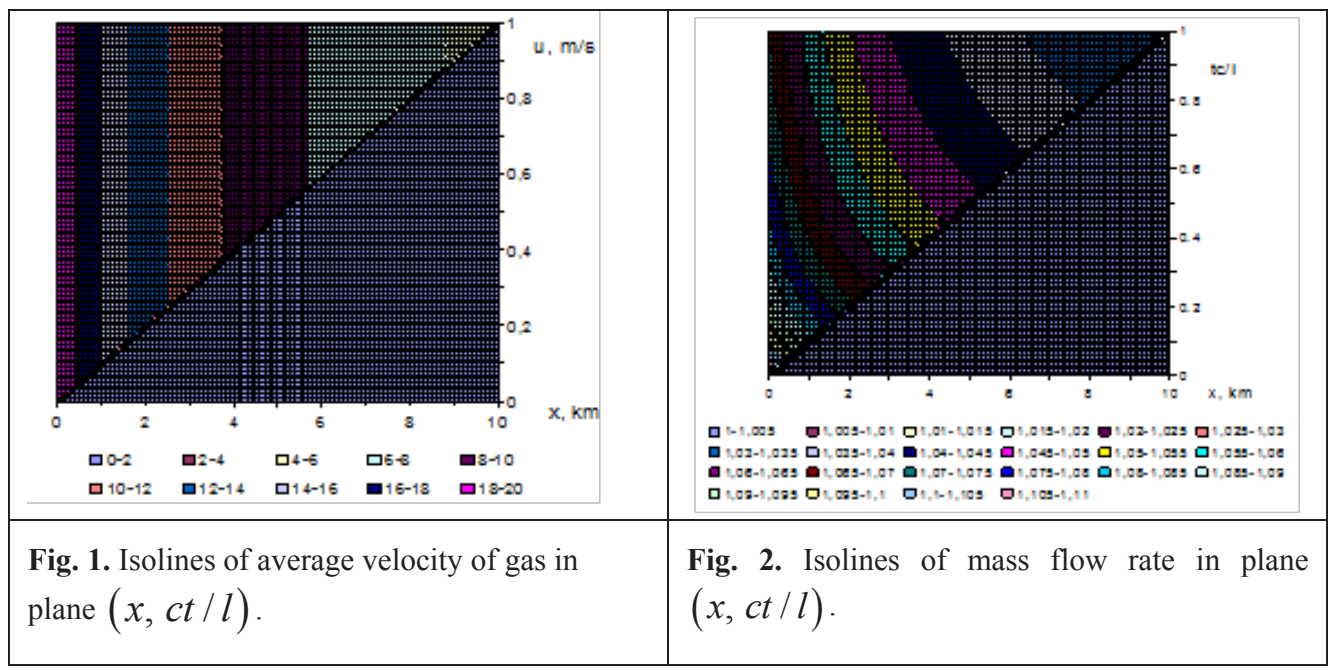

\section{Conclusions}

The paper proposes a mixed method for studying the wave flow of gas in a semi-infinite pipeline. In contrast to the known problems, the propagation of a unilateral wave is considered. The conditions for the uniqueness of the solution to the problem for the gas velocity are taken in the form of one boundary and two initial conditions.

With the introduction of an auxiliary function in the form of the natural logarithm of the reduced density and with the use of gauge functions, the equations are presented in a simpler form concerning the reference solution of the problem. The reference solution was refined by reducing it to the Riemann problem, but taking into account the quadratic law of resistance, and an exact solution to the problem concerning the gas flow rate was obtained.

\section{References}

1. Vanchin A.G. Methods for calculating the operating mode of complex trunk gas pipelines. Electronic scientific journal "Oil and Gas Business"., no.4. pp192-214. http://www.ogbus.ru (2014)

2. Vanchin A. G. Determination of the boundaries of application of stationary and non-stationary models of the gas pipeline operation // Oil and Gas Business: electronic scientific journal., (1). pp 598-617. http://www.ogbus.ru. (2014)

3. Herra'n-Gonza'lez A., De La Cruz J.M., De Andre's-Toro B., Risco-Martı'n J.L. Modeling and simulation of a gas distribution pipeline network // Applied Mathematical Modelling, 33 pp1584-1600. http://dx.doi.org/10.4236/jamp.2016.48175. (2009).

4. Leugering Günter. Domain Decomposition of an Optimal Control Problem for Semi-Linear Elliptic Equations on Metric Graphs with Application to Gas Networks // Applied Mathematics, , 8. - pp. 1074-1099 http://www.scirp.org/journal/am. (2017).

5. Pankratov S. N., Kazak A. S., Chionov A. M., Kazak K. A., Kireev A. Yu., Morgunov A. A. Gas quality control on long underwater high-pressure gas pipelines. - Transport of gas and gas condensate 8 (756), pp. 74-79. (2017).

6. Wang Hai, Liu Xiaojing, Zhou Weiguo. Transient flow simulation of municipal gas pipelines and networks using semi implicit finite volume method // 2011 SREE Conference on Engineering Modelling and Simulation (CEMS 2011) Procedia Engineering 12 pp 217-223. (2011). 
7. Neidorf R. A., Teterevleva E. V., Yagubov Z. Kh. Calculation of reference parameters of transported gas flows for problems of simulation. - Bulletin of DSTU, 4 (55). pp. 470-479. (2011).

8. Штыков Р. А. Прочесс изменения коэффициента сверхсжимаемости газа на участках газопровода // Надежность и качество сложных систем, 1(21). pp 56-63. DOI: 10.21685/2307-4205-2018-1-7. (2018).

9. Сулейманов В. А. Неравновесная термодинамика процесса трубопроводного транспорта природного газа. - Научно-технический сборник: Вести газовой науки. 2 (39), pp 74-79. (2019).

10. Булович С. В. Математическое моделирование течения газа в окрестности открытого ториа трубы при колебаниях пориня на другом конце трубы по гармоническому закону на резонансной частоте, Журнал технической физики, 87, (11). pp 1632-1636. DOI: 10.21883/JTF.2017.11.45121.2086. (2017).

11. Oosterkampa A., Helgakera J. F., Ytrehusb T. Modeling of natural gas pipe flow with rapid transients-case study of effect of ambient model // Energy Procedia. 64,. pp 101-110. (2015).

12. Charny I.A. Unstable motion of real liquid in pipes. 2nd ed. - M.: Nedra, (1975).

13. Seleznev V.E., Aleshin V.V., Pryalov S.N. Modern computer simulators in pipeline transport. Mathematical modeling methods and practical application / Ed. By V.E. Seleznev. - M.: MAKS Press,. p 200. (2007).

14. Samarskiy A.A., Popov Yu.P. Difference schemes of gas dynamics. - M.: Nauka,. p 352. (1975).

15. Bozorov O.Sh., Mamatkulov M.M. Analytical studies of nonlinear hydrodynamic phenomena in media with slowly varying parameters. - Tashkent, TITLP,. p 96. (2015).

16. Tikhonov A.N., Samarskiy A.A. Equations of mathematical physics. - M.: Nauka,. p 736. (1977). 